$\Rightarrow$ IN THE NEWS

\section{New eyes on the Universe}

This month marks the start of the Dark Energy Spectroscopic Instrument (DESI) survey, which will be mapping the distance to 35 million galaxies and 2.4 million quasars across one-third of the sky over the next 5 years. The expected $100 \mathrm{~TB}$ of data per year gathered by the survey is anticipated to

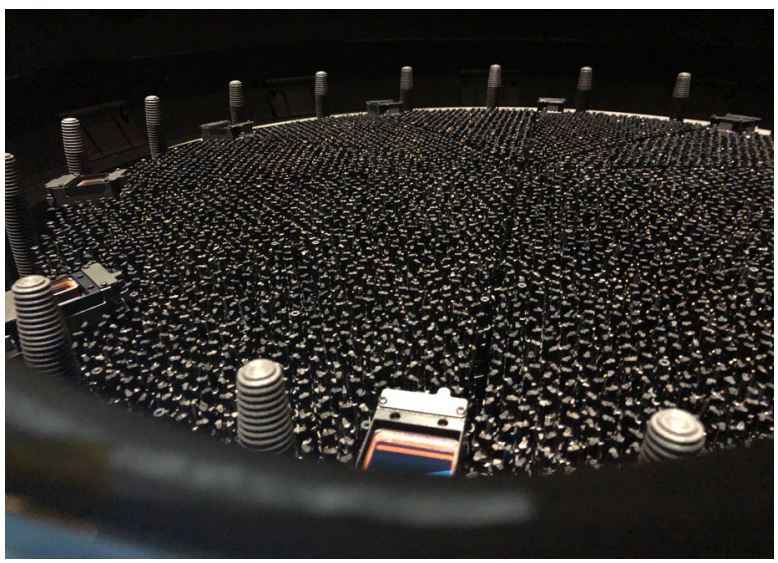

Credit: DESI Collaboration $\odot 2019$ The Regents of the University of California, Lawrence Berkeley National Laboratory improve our understanding of dark energy, the mysterious force driving the accelerated expansion of our Universe.

DESI is mounted on the $4 \mathrm{~m}$ Mayall Telescope at Kitt Peak National Observatory in Arizona. It is a complex instrument made of six large fused silica lenses to provide a larger field of view, a focal system and 10 spectrographs. The focal plane consists of 5,000 robotic positioners (pictured) each holding a $100 \mu \mathrm{m}$ diameter optical fibre. The fibres are gathered into 10 groups of 500, each group feeding into one spectrograph. The robotic positioners automatically rearrange themselves in such a way that light is collected from a certain set of galaxies and quasars. This rearrangement only takes $10 \mathrm{~s}$, providing DESI with unprecedented surveying speed and hence the ability to map 20 times more objects than was possible with previous instruments.
"The individual elements of DESI are complex in their own regard, but more so in the full assembly of the instrument. It has been wonderful to see the whole instrument come together after years of design and construction," says Daniel Eisenstein co-spokesperson of DESI.

To learn more about dark energy, DESI will primarily measure baryon acoustic oscillations - baryonic matter density fluctuations caused by the primordial acoustic density waves - and redshift space distortions, the modifications in the spatial distribution of galaxies caused by the gravitational pull from the surrounding large-scale structure of the Universe. These should improve our understanding of the expansion history of our Universe. Beyond cosmology, DESI is expected to enhance our understanding of the physics of galaxies, quasars and intergalactic gas. With a planned public data release every year there is a lot of excitement in store for cosmologists and astrophysicists.

Iulia Georgescu 